\title{
Transaortic Transcatheter Aortic Valve Implantation: Learning Curve, Perioperative, and Midterm Follow-Up Results of a Single Center
}

\author{
Giuseppe D'Ancona, $\mathbf{M D}, \mathbf{P h D},{ }^{1,2}$ Huesyin Ince, $\mathrm{MD}, \mathrm{PhD},{ }^{1,2}$ Christoph Raspé, $\mathrm{MD}, \mathrm{PhD},{ }^{3}$ \\ Alper Öner, MD, PhD, ${ }^{1,2}$ Evren Caglayan, MD, PhD, ${ }^{1,2}$ Erdal Safak, MD, ${ }^{1,2}$ Hasan Bushnaq, MD ${ }^{4}$ \\ ${ }^{1}$ Vivantes Klinikum im Friedrichshain und Am Urban, Berlin; ${ }^{2}$ Rostock University Medical Center, Rostock; ${ }^{3}$ Department of \\ Anesthesiology and Critical Care Medicine, Halle-Wittenberg University, Halle; ${ }^{4}$ Department of Cardiac Surgery, University \\ Hospital Rostock, Rostock, Germany
}

\section{ABSTRACT}

Background: We present our initial institutional experience with transaortic (TAo) transcatheter aortic valve implantation (TAVI) using a self-expanding aortic bioprosthesis.

Methods: A total of 106 patients underwent TAo TAVI with Medtronic CoreValve through a small partial upper sternotomy. We focus our analysis on the overall perioperative results, procedural learning curve (first 30 patients), and midterm follow-up outcomes.

Results: VARC-2 device success was achieved in 95 patients (89\%), and there were no intraoperative deaths. Nine patients $(8.4 \%)$ required a second valve and conversion to standard surgery was required in 2 patients (1.8\%). The final aortic insufficiency was grade 0 in 65 patients $(62 \%)$ and grade 1 in $39(37 \%)$. Although patients treated in the TAo TAVI learning phase required a significantly longer radiation time and contrast agent use, device success $(93.4 \%$ versus $88.2 \%$; $P=.7)$ and prostheses hemodynamics were similar. All-cause mortality at 30 days was $12 \%(13 / 106)$. At a median followup of 392 days (IQR: 216-494 days) estimated overall 1-year survival was $72 \%$. No significant differences were reported in terms of 30-day and 1-year observed mortality, and estimated 1 -year survival in the learning and later phase of TAo TAVI.

Conclusion: TAo TAVI can be performed safely even in the very early phase of the learning curve. Although satisfactory results can be achieved from the beginning, a significant reduction in contrast agent use and radiological exposure are expected as the technique is mastered. Good hemodynamics have been documented and should be further improved with modifications achieved in the TAVI self-expandable valves technology.

\section{INTRODUCTION}

Transcatheter aortic valve implantation (TAVI) has been proposed and popularized to treat symptomatic severe aortic

Received October 10, 2019; received in revised form fanuary 7, 2019; accepted fanuary 9, 2019.

Correspondence: Giuseppe D'Ancona, MD, PbD, Vivantes Klinikum, Landsberger Allee 49, 10249 Berlin, Germany; +49-30-130-231174; fax: +49 (0)30-130-232063 (e-mail: rgea@hotmail.com). valve stenosis (AVS) in selected patients at increased risk for conventional aortic valve replacement (AVR) on cardiopulmonary bypass (CPB) [Leon 2010; Smith 2011; Popma 2014]. TAVI with a transfemoral (TF) first approach has been used in most treating centers and should be the advocated strategy for the majority of referred patients. Although the ventricular trans-apical route is the one so far mainly used when TFTAVI is not feasible, alternative accesses have been adopted in the clinical practice to limit the surgical invasiveness. In this context, the transaxillary, transcarotid, and transaortic (TAo) approaches have all been proposed as valid alternatives to $\mathrm{TF}$ TAVI [Thourani 2015a].

The cumulative experience with the TAo approach for TAVI using both self-expanding and balloon expandable prosthesis has shown encouraging results regarding feasibility, safety, and overall outcomes [Amrane 2017; Thourani 2015b; Bapat 2016; Bruschi 2015; Reardon 2014; Fröhlich 2015]. Clinical data in single-center everyday practices are still limited, as well as information concerning the learning curve of TAo and its follow-up outcomes.

For this reason, in the present manuscript, we summarize our initial institutional experience with TAo TAVI using a self-expanding aortic bioprosthesis. We focus our analysis on the overall results, procedural learning curve, and midterm follow-up outcomes.

\section{METHODS}

\section{Patients and Procedures}

All patients were treated at the University Heart Center Halle/Saale, Germany, for symptomatic severe AVS from September 2012 through August 2014. Patients underwent TAVI with the Medtronic CoreValve self-expanding prosthesis (Medtronic, Minneapolis, MN, USA) using TAo access. All procedures were performed by the same senior surgeon $(\mathrm{HB})$ or under his strict supervision.

The standard screening process included transthoracic and/or transoesophageal echocardiography (TTE/TEE), coronary angiography, and ECG gated multidetector computed tomography (MDCT) of the aortic valve, entire aorta, and iliofemoral vessels. The screening was aimed at defining the anatomy and geometry of the aortic unit and excluding 
Table 1. Preprocedural Data

\begin{tabular}{|c|c|c|c|c|}
\hline Age, y & $80.3 \pm 6.2$ & $79.4 \pm 7.3$ & $80.3 \pm 5.4$ & .5 \\
\hline STS score & 6.6 (IQR: 3.5-12.9) & 7.3 (IQR: 3.6-10.7) & 6.0 (IQR: 3.6-9.2) & .2 \\
\hline $\mathrm{BMI}, \mathrm{kg} / \mathrm{m}^{2}$ & $27.2 \pm 4.5$ & $28.4 \pm 4.3$ & $26.8 \pm 4.4$ & .09 \\
\hline Mean TransAo gradient, $\mathrm{mmHg}$ & 41.0 (IQR: 30.0-49.2) & 39.0 (IQR: 29.0-45.5) & 42.0 (IQR: 31.2-52.2) & .6 \\
\hline Annulus diameter at CT & $25.8 \pm 9.9$ & $25.5 \pm 10.0$ & $25.9 \pm 9.9$ & .8 \\
\hline
\end{tabular}

STS indicates Society of Thoracic Surgeons; BMI, body mass index; LVEF, left ventricular ejection fraction; TEE Aortic EOA, transesophageal echocardiography aortic effective orifice area; CT, computed tomography.

the presence of significant atherosclerotic disease of the ascending aorta.

In the present series, TAo TAVI was the chosen approach independently by the quality of the femoro-iliac vasculature. The decision for the access type was discussed and finalized by a dedicated multidisciplinary heart team, including the cardiologist and heart surgeon.

As for institutional policy, TAo TAVI is performed under general anesthesia, endotracheal intubation, and mechanical ventilation. A temporary pacemaker is placed in the right ventricle through the femoral vein to perform rapid ventricular pacing whenever required and as a back-up to treat complete atrioventricular block after TAVI.

Most of the surgical aspects of the TAo TAVI have been already clarified in the previous literature.

A partial upper sternotomy is the favored incision in our practice for the majority of patients. This incision is the one that most resembles the standard approach used for surgical AVR. After spreading the sternal edges with a minithoracotomy retractor, the aorta is identified, and digital palpation is used to confirm the absence of atherosclerotic plaques.

At this stage, an ascending aorta aortography is performed with a graduated pigtail to define the exact position of the entry site in relation to the aortic annulus. In fact, having the CoreValve a total stent length of $5.5 \mathrm{~cm}$, the aortic entry site should be located at least at $6 \mathrm{~cm}$ from the aortic annulus. After opening the upper third of the pericardium, the pericardial edges are suspended, and two aortic purse-string sutures are placed at the aortic entry site.

The remaining parts of the procedure, including aortic cannulation, aortic balloon valvuloplasty, and TAVI are performed as previously described [Amrane 2017; Thourani 2015b; Bapat 2016; Bruschi 2015; Reardon 2014; Fröhlich 2015].

\section{Data Collection and Statistical Analysis}

All patients gave signed informed consent for treatment and use of their data for scientific research purposes. The study was approved by the local research committee, and no ethical committee evaluation was required, as the procedures and additional treatments described in the study are part of our clinical standard of practice.

After treatment and discharge, patients underwent a telephone based follow-up. Data were collected prospectively and analyzed retrospectively. Data are presented as rates for categorical variables and mean with standard deviation (normally distributed variables) or median with $75 \%$ interquartile ranges (IQR) (not normally distributed variables) for continuous variables. Variables normality has been tested by means of Wilk-Shapiro test.

To identify the possible impact of learning curve upon outcomes, two groups were identified. The first 30 TAo TAVI patients were considered as learning curve patients, in accordance with previously published evidence on patients undergoing transapical TAVI [Suri 2016].

Differences between the two groups were tested by means of unpaired Student $t$ test, Mann-Whitney test, Pearson chisquare test, and Fischer exact test whenever appropriate.

Moreover, the effect of the learning curve was assessed by the time effectivity of the procedure focusing on operating time, contrast medium use, and fluoroscopy time. Changes during the study period were correlated with the consecutive number of the procedure (Spearman rank test). Linear regression was used to quantify the trends over time. Finally, Kaplan-Meier curves for survival were built, and estimated rates were reported for the overall cohort. Moreover, equality of survival distribution between patients belonging to the learning and later treatment phases was tested (Mantel-Cox, Breslow, Tarone-Ware).

The statistical calculations were run using the SPSS 11.0 software (SPSS for Windows, Chicago, SPSS).

\section{RESULTS}

\section{Patients}

A total of 106 patients underwent TAo TAVI with Medtronic CoreValve. The demographic and baseline 
Table 2. Periprocedural Data

\begin{tabular}{|c|c|c|c|c|}
\hline Valve 26 mm, n (\%) & $54(51)$ & $10(33)$ & $44(58)$ & .04 \\
\hline Valve 31 mm, n (\%) & $11(10)$ & $1(3)$ & $10(13)$ & \\
\hline Second valve implanted, n (\%) & $9(8)$ & $3(10)$ & $6(8)$ & .8 \\
\hline Contrast agent, $\mathrm{mL}$ & $120.6 \pm 56.2$ & $171.4 \pm 46.2$ & $99.3 \pm 45.1$ & $<.0005$ \\
\hline Fluoroscopy time, $\min$ & $14.2 \pm 8.1$ & $20.7 \pm 9.4$ & $11.8 \pm 5.4$ & $<.0005$ \\
\hline VARC-2 device success, $\mathrm{n}(\%)$ & $95(89)$ & $28(93)$ & $67(88)$ & .7 \\
\hline Mean Trans-Ao gradient, $\mathrm{mmHg}^{*}$ & $4.3 \pm 2.4$ & $5.0 \pm 2.1$ & $4.0 \pm 2.5$ & .06 \\
\hline Final Al 0, n (\%)* & $65(62)$ & $22(73)$ & $43(58)$ & .4 \\
\hline
\end{tabular}

*Two patients converted to standard aortic valve replacement are excluded.

VARC indicates Valve Academic Research Consortium; Al, aortic insufficiency.

clinical characteristics are summarized in Table 1. Although the cohort had a highly comorbid profile, as demonstrated by the high median logistic EuroSCORE of 16.4\% and median Society of the Thoracic Surgeons predicted risk of mortality score (STS-PROM) of 6.6\%, severe peripheral vascular disease (PVD) limiting a possible TF access was present in only 36 patients (34\%).

Moreover, Table 1 shows no significant difference in the demographic and baseline surgical risk profile of the learning curve (first 30 TAo TAVI patients) and later phase TAo TAVI patients (76 patients).

\section{Perioperative Outcomes}

Procedural characteristics and composite endpoints according to Valve Academic Research Consortium (VARC) are presented in Table 2. VARC-2 device success was achieved in 95 patients (89\%), and there were no intraoperative deaths. Partial upper sternotomy was performed in 100 patients (94\%) and minithoracotomy in one patient (1\%). Five patients $(5 \%)$ underwent concomitant off-pump CABG and TAo TAVI through a full sternotomy.

All patients underwent an aortic balloon valvuloplasty with a non-compliant balloon before TAVI.

Nine patients $(8.4 \%)$ required a second valve: $3(10 \%)$ in the early phase and $6(7.8 \%)$ in the later phase $(P=.7)$ of the TAo experience. A second prosthesis of the same size (5 patients) or a larger size (4 patients) was used to treat persistent moderate to severe paravalvular leak. No valve migrations were reported.

Conversion to standard AVR was required in 2 patients $(1.8 \%)$, in one patient to treat the consequences of acute coronary occlusion and in one patient to manage persistent severe aortic regurgitation. Both conversions occurred in the later phase of TAo experience.

Post-dilatation due to more than moderate aortic regurgitation was necessary in 8 patients $(7.5 \%)$. Excluding the two patients undergoing conversion to surgical AVR on CPB, the final AI at TEE was grade 0 in 65 patients $(62 \%)$ and grade 1 in $39(37 \%)$.

Although patients treated in the TAo TAVI learning curve phase required a significantly longer radiation time and contrast agent use, device success $(93.4 \%$ versus $88.2 \% ; P=.7)$ and prostheses hemodynamics were similar in the two groups (Table 2).

Using Spearman test, there was no significant correlation between TAo TAVI overall implantation time and experience $(P=.2)$. Fluoroscopy time and contrast agent amount decreased significantly during the study period (rho $=-0.5$, $P<.0001$ and rho $=-0.7, P<.0001$ respectively). At linear regression per every treated patient, there was a significant 7.5 seconds reduction in fluoroscopy time $(\mathrm{B}=-7.5$, CI: $-10.1 /$ $5.0 ; P<.0001)$ and a decrease of $1.2 \mathrm{~mL}$ in used contrast agent $(\mathrm{B}=-1.2$, CI: $-1.4 /-0.9 ; P<.0001)$.

\section{Clinical Outcomes}

Table 3 summarizes 30-day morbidity and mortality.

The incidence of access site (major vascular) complications was $3.7 \%$ (4/106 patients), and no aortic dissection was observed. Life-threatening bleeding occurred in $13 \%$ of patients $(\mathrm{n}=14)$, whereas the median amount of transfused packed red blood cells was 1.0 (IQR: 0-2 units). Acute kidney injury developed in 20 patients (19\%), though stage 2 and 3 injury was present in $7(6.6 \%)$ and $2(1.8 \%)$ patients. Myocardial infarction occurred periprocedurally in one case (1\%) due to occlusion of the left coronary ostium by plaque shift (conversion to surgical AVR). One patient (1\%) suffered from a major cerebral stroke. New pacemaker implantation was required in 29 cases $(27 \%)$.

In synthesis, the 30-day VARC-2 early safety endpoint was reached overall in 35\% (37/106) of the TAo patients, more specifically in 7 of the learning phase (23.3\%) and $30(39.5 \%)$ of the late 
Table 3. 30-day Morbidity and Mortality

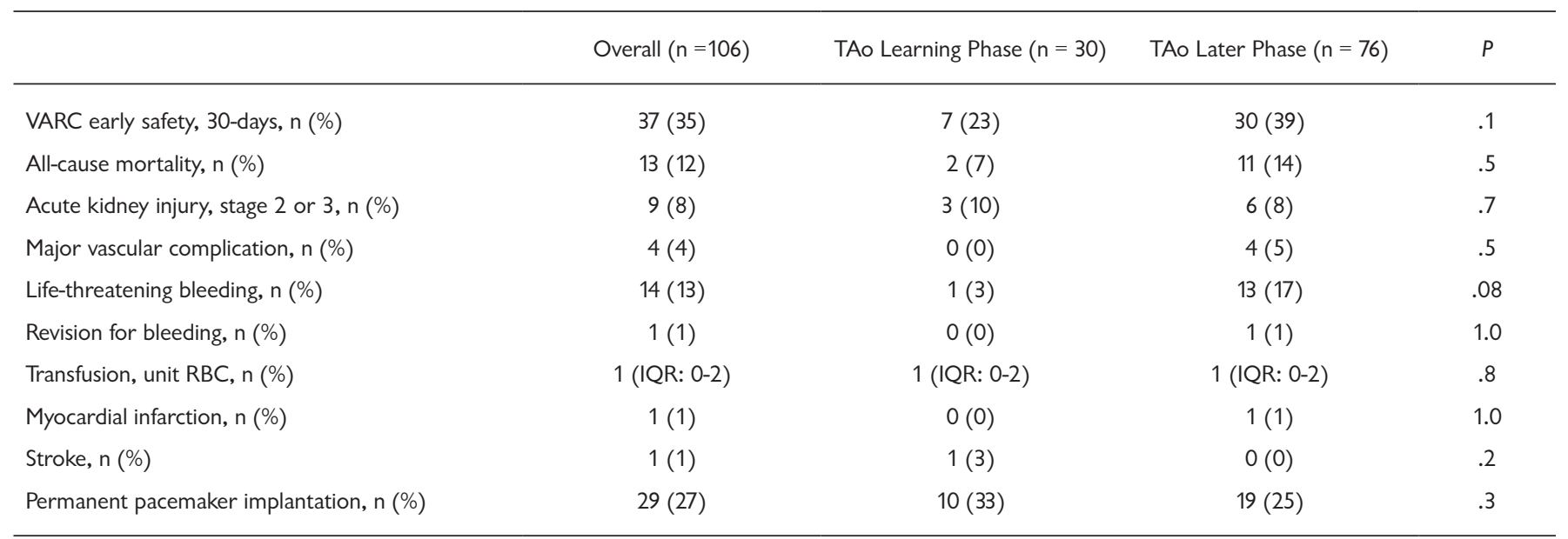

VARC indicates Valve Academic Research Consortium; PRBC, red blood cells.

phase $(P=.1)$. All-cause mortality at 30 days was $12 \%(13 / 106)$ (TAo learning phase $7 \%$ versus TAo later phase $14 \% ; P=.5$ ).

Specific complications rates were similar in the learning and later phase of TAo TAVIs.

\section{Follow-Up Outcomes}

All surviving patients were discharged either to a rehabilitation center or home. One-year cardiovascular-related rehospitalization was observed in 10 patients $(9.4 \%)$. No patient required additional intervention on the aortic valve at midterm follow-up. Overall mortality at one year was $27 \%$ (29/106) and cardio-vascular related death occurred in 14 patients (13.2\%). At a median follow-up of 392 days (IQR: 216-494 days) estimated overall 1-year survival was $72 \%$.

No significant differences in terms of 1-year observed mortality (learning phase $24.1 \%$ vs later phase $28.9 \%$; $P=.6$ ) and 1-year estimated survival (learning phase $76 \%$ versus later phase $71 \% ; P=.5$ ) were reported between the learning and later phase TAo TAVI patients (Figure).

\section{DISCUSSION}

The present study represents possibly one of the largest single-center experiences with TAo TAVI and gives some new insights in terms of learning curve and midterm outcomes of this underused approach.

These patients were treated in a very early TAVI phase when the real benefits and feasibility of a mainly TF approach were not yet clear. At that time TAo TAVI was considered as a possible and reasonable approach independently by the quality of the femoro-iliac vasculature. In the present report severe PVD was present in only $34 \%$ of the patients and most of the treated patients could have been managed also using a TF approach.

TAVI operators are nowadays aware that the TF approach is the gold standard. In fact, there are a limited number of
TAVI candidates that, at least with the present technology, cannot be treated using the TF approach. Consequently, treating physicians have few opportunities to master alternative surgical approaches for TAVI.

While satisfactory results have been shown when using alternative approaches for TAVI, a learning curve should be expected before steady-state results can be achieved. In this context, the TAo route may represent the most familiar and easily learnable approach for the majority of surgeons for two main reasons: type of incision (sternotomy) and type of vascular access (ascending aorta cannulation) are part of the daily routine of every practicing cardiac surgeon. To further simplify the procedure, in our experience we have mainly adopted an upper partial sternotomy to guarantee for optimal distance between sheath entry point and native aortic valve annulus. Using this incision, the aorta immediately proximal to the brachiocephalic trunk can be reached. At this level, the aortic wall quality is often better than that observed in the more proximal post-stenotic and dilated ascending aorta site.

In a recent meta-analysis Amrane et al [Amrane 2017] showed that the reporting of TAo TAVI remains quite limited with not even 2000 cases documented in the existing literature. Although no specific investigation concerning the learning curve of TAo TAVI has been so far conducted, results seem to be homogenous independently by center experience (ranging from 13 to 94 patients for single-center studies), used surgical incision (partial mid-sternotomy or small-thoracotomy), and adopted TAVI prosthesis (self-expandable or balloon expandable) [Amrane 2017].

Although the majority of published TAo experiences have not reported results according to VARC criteria, the average device success rate of $91 \%$ and 30 -day early safety rate of $16 \%$ are encouraging and comparable to those achieved with the TF approach [Amrane 2017].

This could be due to the fact that, as already emphasized, cannulation of the ascending aorta is mastered by every practicing cardiac surgeon and direct exposure of the vessel 


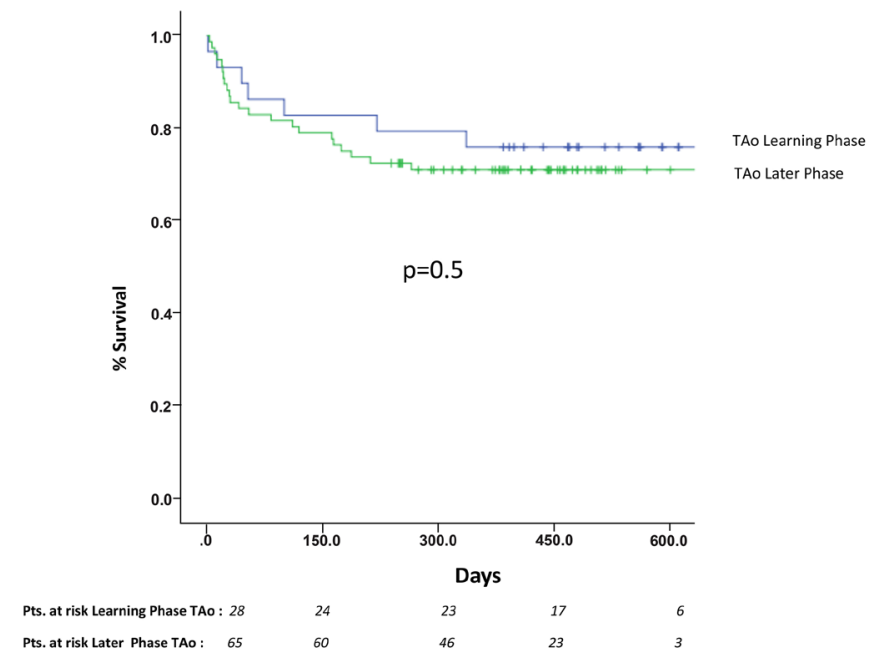

Follow-up survival curves in patients undergoing TAo TAVI.

facilitates immediate and agile management of local complications. As a result, the vascular complication rate of TAo TAVI is $3 \%$ on average [Amrane 2017]. This satisfactory result has been achieved even in the earlier phases of TAo TAVI while adopting first generation TAVI prostheses that were loaded on higher profile sheaths. In the same context, both PARTNER trials (A and B) have reported a vascular complication rate of TF-TAVI over $10 \%$ with a direct relationship between short-term mortality and vascular complications [Leon 2010; Smith 2011].

Precise prosthesis positioning within the selected landing zone is one of the prerogatives to achieve adequate valve hemodynamics during TAVI. The vicinity between the ascending aorta entry point and the native aortic annulus guarantees for a controlled and timely prosthesis positioning that results in a low rate of residual severe paravalvular leak (6.7\% on average) [Amrane 2017]. These results have to be interpreted in light of the fact that most of the reported TAo experiences (like the one herein presented) have included mainly patients treated with first-generation TAVI prosthesis where features of optimized valve sealing and prosthesis resheathability/repositionability were not yet available.

As confirmed in our findings the TAo TAVI learning curve is not steep and, after a contained number of patients, radiation exposure and contrast agent use are significantly reduced, and continue to decrease as operator experience increases.

We tested a learning curve cut-off of 30 patients that was already proposed in a sub-group analysis of the PARTNER-I trans-apical TAVI patients [Suri 2016]. It is noteworthy that none of the patients treated in the earlier phase of our experience required conversion to standard AVR and/or cardiopulmonary bypass support. Moreover, since the beginning of the TAo experience, device success was over $90 \%$. Whenever necessary, implantation of a second valve occurred in $10 \%$ of the patients that were treated in the early phase. Implantation of a second valve was mainly adopted to manage severe residual paravalvular leak after final complete release of a first generation self-expandable and non-re-sheathable TAVI prosthesis. This may have led to a rate of second valve implantation higher than expected. Finally, none of the patients presented here experienced residual paravalvular regurgitation greater than mild at discharge. These results are expected to be further optimized since the recent introduction of fully repositionable and resheathable self-expandable TAVI devices that can also be implanted through the TAo route.

As already noted, the 30-day VARC early safety of TAo TAVI has seldom been reported (in only $50 \%$ of the existing studies) and has been ranging between $9 \%$ and $31 \%$ with a pooled estimated value of $16.7 \%$ [Amrane 2017]. Our proposed value of $35 \%$ within our global experience may seem in this context very high. Interestingly, at parity of patients risk profile, 30-day adverse events were less common in the early phase of the TAo experience. In any case, most of the early safety events reported in our experience have been categorized as major bleeding according to VARC criteria (BARC 3a). In most of these cases, in fact, there was a decrease in hemoglobin of at least $3 \mathrm{gr} / \mathrm{dL}$ during the procedure. This was mainly the result of extreme perioperative hemodilution. Blood transfusion rate was contained, and only one patient required revision for surgical bleeding.

In hospital 30-day mortality of TAo TAVI is within the ranges observed with the TF approach. Amrane et al calculated in their meta-analysis a pooled value of 9.9\% [Amrane 2017]. Our reported 30-day mortality rate is in line with the values proposed in the literature, and it has remained contained since the beginning of our experience with TAo TAVI.

Follow-up outcomes after TAo TAVI have seldom been investigated. In the alternative access arm of the CoreValve US Pivotal Trial, Reardon et al documented a 36\% one-year overall mortality rate for TAVI patients treated using either the trans-subclavian or TAo approach [Reardon 2014]. This value is slightly higher than the $26 \%$ all-cause one-year mortality rate observed in the TF cohort of the same trial [Popma 2014]. In a series of 100 TAo TAVI patients treated with an expandable balloon prosthesis, Petzina et al documented long-term mortality of 38\% [Petzina 2017]. A 30.3\% oneyear mortality rate has been documented in a US multicenter experience with TAo TAVI using a balloon expandable device [Thourani 2015; Thourani 2015b].

Data from the UK TAVI registry suggest transapical and TAo TAVI to be associated with similar survivals that are both significantly worse than those observed with the TF and trans-subclavian routes [Fröhlich 2015].

Our $27 \%$ one-year all-cause mortality is comparable to those proposed in other TAo experiences, but it is slightly higher than those documented in real-world registries of TF TAVI with the same first generation self-expandable prosthesis [Linke 2014; Barbanti 2015].

In the ADVANCE study, Linke et al documented a 12 -month overall mortality rate of $17.9 \%$ [Linke 2014]. Similarly, Barbanti et al published a 21\% one-year overall mortality in the Clinical Service Project [Barbanti 2015].

In reality, we observed that more than half of our follow-up deaths occurred for non-cardiovascular reasons. In this context, our one-year cardiovascular mortality of $13.2 \%$ is more in line 
with the values proposed in the ADVANCE $(11.7 \%)$ and Clinical Service Project (10\%) [Linke 2014; Barbanti 2015].

In any case, when we strictly focus upon the impact of the learning curve in TAo TAVI, we clearly see that even midterm survival is not influenced by the learning curve. In fact, estimated one-year survival remains similar in the early and later phase patients treated with TAo TAVI.

\section{Conclusion}

As the boundaries of TF TAVI are expanding, operators have a limited possibility to master alternative approaches for TAVI. In this context the TAo approach presents some characteristics that could make it more suitable to the standard cardiac surgery practice, whenever required.

We confirm that TAo TAVI can be performed safely even in the very early phase of the learning curve. Although satisfactory results can be achieved from the beginning, a significant reduction in contrast agent use and radiological exposure are expected as the technique is mastered with the increasing in experience. Good hemodynamic performances of the implanted TAVI prostheses have been documented and should be further improved with the recent modifications achieved in the TAVI self-expandable valves technology. Midterm follow-up outcomes are encouraging and in line with those observed when using the TF approach.

\section{REFERENCES}

Amrane H, Porta F, Van Boven AV, Kappetein AP, Head SJ. 2017. A meta-analysis on clinical outcomes after transaortic transcatheter aortic valve implantation by the Heart Team. EuroIntervention 13:e168-e176.

Bapat V, Frank D, Cocchieri R, et al. 2016. Transcatheter aortic valve replacement using transaortic access: experience from the Multicenter, Multinational, Prospective ROUTE Registry. JACC Cardiovasc Interv 9:1815-22.

Barbanti M, Petronio AS, Ettori F, et al. 2015. 5-Year outcomes after transcatheter aortic valve implantation with CoreValve prosthesis. JACC Cardiovasc Interv 8:1084-91.
Bruschi G, De Marco F, Botta L, et al. 2015. Right anterior mini-thoracotomy direct aortic self-expanding trans-catheter aortic valve implantation: A single center experience. Int J Cardiol 181:437-42.

Fröhlich GM, Baxter PD, Malkin CJ, et al. 2015. National Institute for Cardiovascular Outcomes Research. Comparative survival after transapical, direct aortic, and subclavian transcatheter aortic valve implantation (data from the UK TAVI registry). Am J Cardiol 116:1555-9.

Leon MB, Smith CR, Mack M, et al. 2010. Transcatheter aortic-valve implantation for aortic stenosis in patients who cannot undergo surgery. N Engl J Med 363:1597-1607.

Linke A, Wenaweser P, Gerckens U, et al. 2014. Treatment of aortic stenosis with a self-expanding transcatheter valve: the International Multicentre ADVANCE Study. Eur Heart J 35:2672-84.

Petzina R, Lutter G, Wolf C, et al. 2017. Transaortic transcatheter aortic valve implantation: experience from the Kiel study. Interact Cardiovasc Thorac Surg 24:55-62.

Popma JJ, Adams DH, Reardon MJ, et al. 2014. Transcatheter aortic valve replacement using a self-expanding bioprosthesis in patients with severe aortic stenosis at extreme risk for surgery. J Am Coll Cardiol 63:1972-81.

Reardon MJ, Adams DH, Coselli JS, et al. 2014. Self-expanding transcatheter aortic valve replacement using alternative access sites in symptomatic patients with severe aortic stenosis deemed extreme risk of surgery. J Thorac Cardiovasc Surg 148:2869-76.

Smith CR, Leon MB, Mack MJ, et al. 2011. Transcatheter versus surgical aortic-valve replacement in high-risk patients. N Engl J Med 364:2187-98.

Suri RM, Minha S, Alli O, et al. 2016. Learning curves for transapical transcatheter aortic valve replacement in the PARTNER-I trial: Technical performance, success, and safety. J Thorac Cardiovasc Surg 152:77380.e14.

Thourani VH, Li C, Devireddy C, Kilgo P, et al. 2015. High-risk patients with inoperative aortic stenosis: use of transapical, transaortic, and transcarotid techniques. Ann Thorac Surg 99:817-23.

Thourani VH, Jensen HA, Babaliaros V, et al. 2015. Transapical and transaortic transcatheter aortic valve replacement in the United States. Ann Thorac Surg 100:1718-26. 